\title{
A Comparative Study of Cervical Composite Restorations Microleakage Using Dental Universal Bonding and Two-step Self-etch Adhesive
}

\author{
Kamyar Fathpour ${ }^{1}$, Amirhosein Bazazzade ${ }^{2}$, Hesam Mirmohammadi ${ }^{3}$
}

\begin{abstract}
Aim: The aim of this study is to compare microleakage of cervical restorations using universal bonding and two-step self-etch adhesive with or without enamel etching through a dye penetration testing method.

Materials and methods: In this in vitro experimental study, 70 maxillary premolar teeth with no anomaly or decay were randomly divided into five groups of 14. Conventional cervical cavities were prepared with diamond bur on the facial surface of the teeth. The teeth were prepared using G-Premio Bond/self-etch technique, G-Premio Bond/total-etch technique, G-Premio Bond/selective-etch technique, Clearfil SE Bond/ self-etch technique, and Clearfil SE Bond/selective-etch technique in Groups I to V, respectively, and restored with composite. Microleakage at the occlusal enamel margin and the cervical dentinal margin was ranked from 0 to 4 based on dye penetration using stereomicroscope $(\times 32)$. Statistical analysis was carried out using Kruskal-Wallis and Mann-Whitney tests at a significance level of $a=0.05$.

Results: The mean occlusal microleakage in the five study groups was $1.07,0.57,0.57,1.28$, and 0.78 , respectively. The mean cervical microleakage was $0.97,0.85,0.64,1.35$, and 1.14 , respectively. Kruskal-Wallis test showed significant difference between the microleakage scores of different groups in enamel $(p=0.022)$ and dentin $(p=0.01)$. Clearfil SE Bond/self-etch technique group showed the highest mean score of microleakage in enamel margins, and G-Premio Bond/total-etch technique and G-Premio Bond/selective-etch technique groups showed lowest enamel microleakage mean. Clearfil SE Bond/self-etch group showed the highest mean score of microleakage in dentin margins, while G-Premio Bond/ selective-etch technique group showed the lowest mean score of dentin microleakage.

Conclusion: Selective etching of enamel will decrease enamel microleakage. G-Premio Bond shows better microleakage results in comparison to Clearfil SE Bond in dentinal margins.

Clinical significance: A major problem in cervical composite restorations is the presence of microleakage in the cervical wall. Different adhesive systems have been designed to overcome this problem.

Keywords: Microleakage, Selective enamel etch, Self-etch bonding, Universal bonding

The Journal of Contemporary Dental Practice (2021): 10.5005/jp-journals-10024-3171
\end{abstract}

\section{INTRODUCTION}

One of the remarkable progresses in restorative dentistry is the technology of resin-based composites. ${ }^{1}$ Composite restorations have certain advantages over amalgam restorations including preservation and adhesion to tooth structure. ${ }^{2}$ Restoration of cervical cavities is one of the indications of composite resins. ${ }^{3,4}$ Microleakage, defined as the diffusion of the bacteria, fluids, molecules, and/or ions into the space between the walls of the cavity and the restorative materials, is a major problem in cervical composite restorations $s^{4,5}$ resulting in marginal discoloration, sensitivity, recurrent caries, pulp irritation, and eventually failure of the restoration. Therefore, it is necessary to prevent microleakage when using an adhesive system. ${ }^{6}$ One of the limitations of cervical lesion is the high C-factor in this restoration that increases the degree of marginal microleakage. ${ }^{7,8}$

Perfect adaptation between restorative materials and cavity margins is necessary for achieving a successful restoration. ${ }^{9,10}$ Currently, dental adhesive systems provide a favorable marginal seal and reduce marginal microleakage in composite restorations. ${ }^{11}$ Many studies have measured the degree of microleakage in cavities restored with dentin bonding systems, but no system has been able to prevent microleakage completely. ${ }^{12}$

There are two strategies for modern dental adhesive systems: Etch-and-rinse systems and self-etch systems with different steps of application. ${ }^{13,14}$ Nowadays, various types of adhesive systems are
${ }^{1}$ Department of Restorative Dentistry, Dental Materials Research Center, Dental Research Institute, Isfahan University of Medical Sciences, Isfahan, Iran

${ }^{2}$ Department of Operative Dentistry, Dental School, Isfahan University of Medical Sciences, Isfahan, Iran

${ }^{3}$ Iranian Center for Endodontic Research, Research Institute of Dental Sciences, Shahid Beheshti University of Medical Sciences, Tehran, Iran; Department of Cariology, Endodontology, Pedodontology, Academic Centre for Dentistry Amsterdam (ACTA), Universiteit van Amsterdam and Vrije Universiteit, Amsterdam, Netherlands

Corresponding Author: Kamyar Fathpour, Department of Restorative Dentistry, Dental Materials Research Center, Dental Research Institute, Isfahan University of Medical Sciences, Isfahan, Iran, Phone: +0098913-310-9432, e-mail: kamyarfathpour@gmail.com

How to cite this article: Fathpour K, Bazazzade A, Mirmohammadi H. A Comparative Study of Cervical Composite Restorations Microleakage Using Dental Universal Bonding and Two-step Self-etch Adhesive. J Contemp Dent Pract 2021;22(9):1035-1040.

Source of support: Nil

Conflict of interest: None

available and there are ongoing studies to simplify and improve the performance of these systems. ${ }^{15}$ Three-step etch and rinse systems 
and two-step self-etch systems have shown decent clinical results in many syudies. ${ }^{13,16,17}$ Different studies show different results of microleakage for adhesive systems. ${ }^{18}$ Some studies have shown that self-etch systems are as effective as etch and rinse systems in preventing microleakage. ${ }^{19,20}$ Nowadays, universal adhesives, which can be used in the self-etch, Total Etch, or Selective-etch technique, are being used. These adhesives can create bond to different substrates including dentin, enamel, glass ionomers, metals, and ceramics. ${ }^{21}$

In a review study by Shroeder et al., ${ }^{22}$ it was concluded that the adhesive strategy, self-etch mode or etch and rinse mode, did not influence the postoperative sensitivity and the retention rates of composite resin in NCCLs in any of the follow-up periods, but less marginal discoloration was found in etch-and-rinse adhesives. In a study by Khosravi et al. ${ }^{23}$ on the degree of enamel microleakage using Clearfil SE Bond, I-Bond, and Scotchbond Multipurpose adhesive systems, it was concluded that the difference in enamel microleakage was not significant when using Clearfil SE Bond in self-etch or Selective-etch mode. Another study by Kermanshah et al. ${ }^{24}$ showed that using Scotchbond Universal in Total-etch mode resulted in lower degrees of microleakage in enamel margins than self-etch mode. Gupta et al. ${ }^{25}$ concluded that there is no difference between degree of microleakage in composite cervical restorations using two step self-etch and Universal bondings.

It seems that adhesive application strategy and chemical composition of the adhesive affect the degree of microleakage in dentin and enamel margins. The aim of this study was to measure and compare the degree of microleakage in enamel and dentinal margins in cervical restorations using Universal bonding and twostep self-etch bonding with or without enamel etching.

\section{Materials and Methods}

The research protocol of this in vitro experimental study was approved by the ethic research committee of Isfahan University of Medical Sciences, Iran (IEC\#IR.MUI.REC.1397.3.119).

\section{Sample Preparation}

Seventy maxillary premolar teeth with no anomaly or decay extracted for orthodontic reasons were used in this study (significance level of $a=0.05$ ).Teeth were cleaned using ultrasonic device (Varios2 Ultrasonic Scaler, NSK, Japan) to remove external debris and stains, stored in $0.5 \%$ thymol solution for 1 week and kept in normal saline to prevent dehydration. Conventional class $V$ cavities, $3 \mathrm{~mm}$ wide, $1.5 \mathrm{~mm}$ deep, and $1.2 \mathrm{~mm}$ height (Fig. 1) were prepared on the facial surface of the teeth parallel to the cementoenamel junction using high-speed $1.2 \mathrm{~mm}$ diamond cylindrical bur (Tizkavan-Iran). The cervical margin of the preparation was located in dentin and the occlusal margin was located in enamel. The cavities were washed with water for 20 seconds and then dried using an air syringe. Each bur was used to prepare four cavities.

\section{Bonding Procedure}

In this study, G-Premio Bond (GC, Tokyo, Japan) was used as universal bonding and Clearfil SE Bond (Kuraray, Tokyo, Japan) as two-step self-etch adhesive. The teeth were assigned to five different groups:

Group I: Use of G-Premio Bond in self-etch mode (GSE): According to the manufacturer's instructions, one layer of G-Premio Bond was

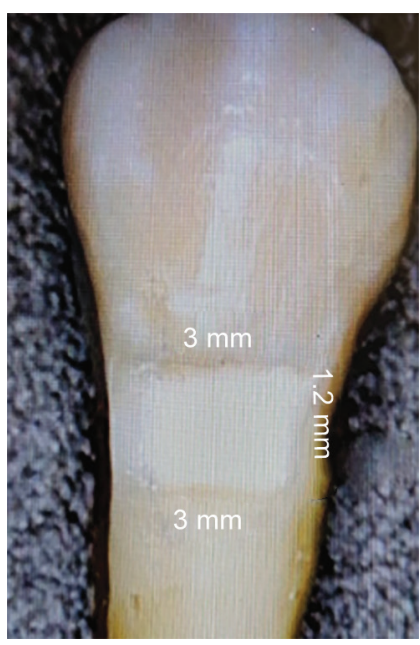

Fig. 1: Prepared cavity dimensions

rubbed onto the cavity surface for 20 seconds with a microbrush. The bond remained on the cavity surface for 10 seconds and the cavity was dried at the maximum airflow rate for 5 seconds. Finally, the adhesive layer was light cured at a light intensity of $800 \mathrm{~mW} / \mathrm{cm}^{2}$ for 10 seconds using VALO Grand device (Ultradent, United States).

Group II: Use of G-Premio Bond in total-etch mode (GTE): 35\% phosphoric acid (Ultradent, United States) was applied to the enamel margins and subsequently to dentin for 15 seconds. After rinsing with air/water spray for 20 seconds, the cavity surface was dried under gentle air pressure until the frosty appearance of the enamel was visible. The procedure of applying G-Premio Bond was similar to Group I.

Group III: Use of G-Premio Bond in selective-etch mode (GSEL): 35\% phosphoric acid was applied to the enamel margins for 20 seconds. After rinsing with air/water spray, the cavity surface was dried under gentle air pressure until the frosty appearance of the enamel was appeared. The procedure of applying G-Premio Bond was similar to Group I.

Group IV: Use of Clearfil SE Bond in self-etch mode (SESE): Following manufacturer's instructions, Clearfil SE Bond primer was rubbed onto the cavity for 20 seconds with a microbrush, the cavity was dried using gentle air pressure for 10 seconds. Subsequently one layer of the Clearfil SE Bond adhesive component was applied onto the cavity with a microbrush and air thinned for 10 seconds using gentle air flow. Finally, the adhesive layer was cured at a light intensity of $800 \mathrm{~mW} / \mathrm{cm}^{2}$ for 10 seconds using VALO Grand device.

Group V: Use of Clearfil SE Bond in selective-etch mode (SESEL): $35 \%$ Phosphoric acid was applied to the enamel margins for 20 seconds. After rinsing with air/water spray for 20 seconds, the cavity surface was dried under gentle air pressure until the frosty appearance of the enamel was appeared. The procedure of applying Clearfil SE Bond is similar to Group IV.

$\mathrm{A}_{2}$ Gradia Direct composite resin (GC, Tokyo, Japan) which is a light-cured microhybrid composite resin was used to restore cervical cavities in three layers on gingival floor, occlusal wall, and the remaining cavity, respectively (Fig. 2). Each layer was light cured at a light intensity of $800 \mathrm{~mW} / \mathrm{cm}^{2}$ for 20 seconds using VALO Grand device. 


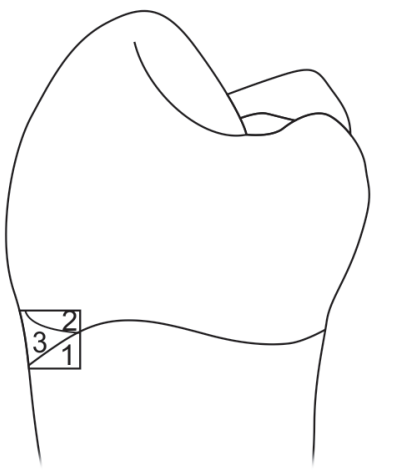

Fig. 2: Layers of composite in cervical cavity

The specimens were finished using flame shape composite resin finishing burs (Tizkavan-Iran). Each bur was used to finish four specimens. The specimens were polished using Soflex polishing disks (3M, Minnesota, United States) which is a four-step composite resin polishing system. Each color coded disk was applied for 5 seconds on the composite surface. Postcuring of the specimens was achieved at a light intensity of $1200 \mathrm{~mW} / \mathrm{cm}^{2}$ for 3 seconds. The specimens were stored in distilled water at $37^{\circ} \mathrm{C}$ for 24 hours. Thermo-cycling was carried out at temperatures of $5 \pm 5^{\circ} \mathrm{C}$ and $55 \pm 5^{\circ} \mathrm{C}$ for 1,000 cycles with a storage time of 30 seconds per cycle.

\section{Microleakage Test}

All specimens were coated with two layers of varnish within a distance of $1 \mathrm{~mm}$ to the margin of the restoration, immersed in $2 \%$ fuchsine at room temperature for 24 hours and washed with distilled water. The specimens were cut buccolingually right from the middle of the restoration using cutting instrument (Vafaei Industrial Co., Tehran, Iran). Microleakage at the occlusal enamel margin and the cervical dentinal margin was observed using a stereomicroscope (10-MBC, HP, United States) at a magnification of $\times 32$ and scored as follows: ${ }^{26}$

0: No microleakage found

1: Dye penetration by one-third of the cavity depth

2: Dye penetration by two-thirds of the cavity depth

3: Dye penetration by more than two-thirds of the cavity depth

4: Dye penetration reaching the axial wall or towards the tooth pulp

Finally, the mean score of microleakage was calculated for each group. Data were statistically analyzed with Kruskal-Wallis and Mann-Whitney tests at a significance level of $a=0.05$.

\section{Results}

Table 1 provides the mean scores of microleakage in five study groups. SESE group showed the highest mean score of microleakage (1.28) and GTE and GSEL groups showed the lowest (0.57) in enamel margins. SESE group showed the highest mean score of microleakage (1.35) and GSEL group showed the lowest score (0.64) in dentinal margins. Table 2 shows the dispersion percentage of occlusal enamel microleakage and Table 3 illustrates the dispersion percentage of cervical dentin microleakage in five study groups.

Kruskal-Wallis test showed that there is a significant difference between the degree of occlusal microleakage $(p=0.022)$ in the
Table 1: Mean scores of microleakage in the study groups

\begin{tabular}{lcc}
\hline Bonding method & Occlusal margin & Cervical margin \\
\hline GSE & $1.07^{(\mathrm{ab})}$ & $0.97^{(\mathrm{cdf})}$ \\
GTE & $0.57^{(\mathrm{a})}$ & $0.85^{(\mathrm{cd})}$ \\
GSEL & $0.57^{(\mathrm{a})}$ & $0.64^{(\mathrm{c})}$ \\
SESE & $1.28^{(\mathrm{b})}$ & $1.35^{(\mathrm{e})}$ \\
SESEL & $0.78^{(\mathrm{a})}$ & $1.14^{(\mathrm{ef})}$ \\
\hline
\end{tabular}

Groups with the same superscript in the same row are not statistically different $(p>0.05)$; GSE, G-Premio Bond in self-etch mode; GTE, G-Premio Bond in total-etch mode; GSEL, G-Premio Bond in selective-etch mode; SESE, Clearfil SE Bond in self-etch mode; SESEL, Clearfil SE Bond in selective-etch mode

five study groups. Also, cervical microleakage difference between five groups was significant ( $p=0.01)$. Based on Mann-Whitney test results, occlusal microleakage was significantly higher in SESE group than GTE $(p=0.009)$, GSEL $(p=0.009)$, and SESEL $(p=0.035)$ groups, while difference between other groups was not significant $(p>0.05)$. Also, Mann-Whitney test showed that cervical microleakage was significantly higher in SESE group than GSE ( $p=0.011)$, GTE $(p=0.008)$, and GSEL ( $p=0.002)$ groups. Comparison of cervical microleakage between SESEL group and the other groups showed that cervical microleakage was significantly higher in this group than GTE $(p=0.05)$ and GSEL $p=0.008)$ groups, while difference between other groups was not significant $(p>0.05)$.

\section{Discussion}

Application of adhesives with separate steps of etching, priming, and bonding resin increases technical sensitivity which is considerably reduced when using self-etch primers that protect smear layer. ${ }^{27,28}$ Strong self-etch systems $(\mathrm{pH}<1)$ etch the smear layer completely similarly to etch-and-rinse adhesive systems, whereas ultra-mild adhesive systems $(\mathrm{pH}>2.5)$ leave the tubules of the smear layer intact. The partial demineralization, which occurs with application of mild self-etch systems $(\mathrm{pH}=2.5)$ (such as Clearfil SE Bond) and ultra-mild self-etch systems, allows for the occurrence of chemical interactions between some functional monomers (such as MDP and 4-META) and hydroxyapatite crystals remaining among collagen fibers. ${ }^{29}$ It seems that the effectiveness of two-step self-etch bonding systems is related to occurrence of chemical and micromechanical bonding. ${ }^{30,31}$

In this study, the significant difference of enamel margin microleakage between SESE group and GTE/GSEL/SESEL groups indicates the importance of acid application on enamel margins for reduction of microleakage. Moreover, the degree of dentinal microleakage was significantly higher in SESE group than in GSE/ GTE/GSEL groups indicating the fact that if acid is not applied prior to application of Clearfil SE Bond, G-Premio Bond shows lower scores of microleakage when used in self-etch, Total Etch, and Selective Etch mode which is due to the specific composition of G-Premio Bond (Table 1).

According to the findings of Hannig et al., ${ }^{32}$ the application of two-step self-etch adhesives on enamel margins creates effective micromechanical bond. Meanwhile, a review by Osorio et al. ${ }^{33}$ shows that no adhesive eliminates microleakage completely. They concluded that Clearfil SE Bond shows lower degrees of microleakage in cervical margins of the restoration. 
Table 2: Different scores of enamel microleakage in the study groups $N(\%)$

\begin{tabular}{lcclccc}
\hline Groups/microleakage score & 0 & 1 & 2 & 3 & 4 & 0 \\
\hline GSE & $4(28.57 \%)$ & $5(35.71 \%)$ & $5(35.71 \%)$ & $0(0 \%)$ & $0(0 \%)$ & $0(0 \%)$ \\
GTE & $6(42.68 \%)$ & $8(57.14 \%)$ & $0(0 \%)$ & $0(0 \%)$ & $0(0 \%)$ & $0(0 \%)$ \\
GSEL & $6(42.68 \%)$ & $8(57.14 \%)$ & $0(0 \%)$ & $0(0 \%)$ & $0(0 \%)$ & 14 \\
SESE & $2(14.29 \%)$ & $6(42.86 \%)$ & $6(42.86 \%)$ & $0(0 \%)$ & 14 \\
SESEL & $3(21.43 \%)$ & $11(78.57 \%)$ & $0(0 \%)$ & $0(0 \%)$ & $0(0 \%)$ & 14 \\
\hline
\end{tabular}

0 , No microleakage found; 1, Dye penetration by one-third of the cavity depth; 2, Dye penetration by two-thirds of the cavity depth; 3, Dye penetration by more than two-thirds of the cavity depth; 4, Dye penetration reaching the axial wall or towards the tooth pulp

Table 3: Different scores of dentin microleakage in the study groups $N(\%)$

\begin{tabular}{lllllll}
\hline Groups/microleakage score & \multicolumn{1}{c}{0} & \multicolumn{1}{c}{1} & \multicolumn{1}{c}{3} & \multicolumn{1}{c}{ Total } \\
\hline GSE & $1(7.14 \%)$ & $13(92.86 \%)$ & $0(0 \%)$ & $0(0 \%)$ & $0(0 \%)$ & 14 \\
GTE & $2(14.29 \%)$ & $12(85.71 \%)$ & $0(0 \%)$ & $0(0 \%)$ & $0(0 \%)$ & 14 \\
GSEL & $5(36.71 \%)$ & $9(64.29 \%)$ & $0(0 \%)$ & $0(0 \%)$ & $0(0 \%)$ & 14 \\
SESE & $0(0 \%)$ & $9(64.29 \%)$ & $5(35.71 \%)$ & $0(0 \%)$ & $0(0 \%)$ & 14 \\
SESEL & $0(0 \%)$ & $12(85.71 \%)$ & $2(14.29 \%)$ & $0(0 \%)$ & $0(0 \%)$ & 14 \\
\hline
\end{tabular}

0 , No microleakage found; 1, Dye penetration by one-third of the cavity depth; 2, Dye penetration by two-thirds of the cavity depth; 3, Dye penetration by more than two-thirds of the cavity depth; 4, Dye penetration reaching the axial wall or towards the tooth pulp

Studies have shown that when phosphoric acid is not used for etching, higher degrees of microleakage are observed in the enamel margins. ${ }^{34,35}$ The present study also shows that the degree of enamel microleakage is significantly lower when Clearfil SE Bond is used in selective-etch mode than self-etch mode. In addition, the degree of enamel microleakage is significantly lower when G-Premio Bond is used in total-etch mode and selective-etch mode than self-etch mode. These significant differences indicate the importance of acid application at enamel margins.

HEMA (hydroxyethylmethacrylate) contained in G-Premio Bond composition may expand dentin collagen fibrils, which in turn leads to increased permeability of the adhesive monomers in the demineralized dentin. ${ }^{36}$ In addition, the water content in G-Premio Bond is approximately $25 \%$, and the $\mathrm{pH}$ of this bond is 1.5. When dentin surface is dried, the water content in the adhesive starts initial ionization of the acidic component and rewets the dried surface of the dentin. Thus, G-Premio Bond is capable of re-expanding the air-dried and collapsed collagen network in order to facilitate the diffusion of resin adhesives. ${ }^{37}$ In addition, the presence of functional monomers such as 4-META, MDP-10, and MDTP in G-Premio Bond may improve bonding performance by interactive effect and increased penetration of monomers into the compressed smear layer. ${ }^{38}$ Therefore, the lower scores of dentinal microleakage with G-Premio Bond in different modes of application compared to Clearfil SE Bond in self-etch mode can be attributed to a better resin adhesive diffusion of monomers into the air-dried and collapsed collagen network. The presence of water and HEMA in the G-Premio Bond adhesive re-expands collagen fibrils which increases permeability of the functional monomers into the demineralized dentin. Hence this bonding can be used in total-etch or self-etch modes. Dentin surface is completely air dried and the collagen collapse will be compensated.

The present study showed that no adhesive system could completely eliminate microleakage. Moreover, the degree of dentinal microleakage was significantly higher in SESE group than GSE, GTE, and GSEL groups (Table 1), indicating that if phosphoric acid is not applied prior to application of Clearfil SE Bond, the microleakage scores of G-Premio Bond will be lower in different modes.

In a systemic review by Szesz et al. ${ }^{39}$ on the effect of selective etching technique for restoring cervical lesions with self-etch adhesives, they concluded that selective etching of enamel margins prior to application of self-etch adhesive systems in noncarious cervical lesions can produce composite restorations with higher longevity. Their review showed that less loss of retention of the restorations at the 3-year follow-up was observed with the selective etching of enamel when using self-etch adhesives. Lower scores of microleakage when using acid in enamel margins in our study are in accordance with this systemic review. In a study by Kearns et al., ${ }^{40}$ a significant difference was observed between the degrees of cervical microleakage when Scotchbond Universal adhesive and Prime and Bond Elect Universal adhesive were used in the self-etch mode and total etch mode but no significant difference was observed between the degrees of cervical microleakage when Futurabond $U$ was used in the self-etch mode and total etch mode. Their study confirms that it is essential to separately evaluate the microleakage of each adhesive system along with their specific formulations and steps. Also, comparing results between present study and studies conducted by Khosravi et al., ${ }^{23}$ Kermanshah and Khorsandian ${ }^{24}$ and Gupta et al. ${ }^{25}$ indicates the importance of adhesive chemical composition and mode of application on the microleakage of the composite restorations.

The main limitation of this study is that microleakage test was done immediately after bonding process and thermocycling. It is necessary to assess microleakage after long period of time. Also it is necessary to assess chemical, mechanical, and biological degradation on microleakage. 


\section{Conclusion}

Given the limitations of this study, the application of phosphoric acid gel prior to bonding process decreases microleakage score for both tested adhesives (G-Premio Bond and Clearfil SE Bond). In dentin margins G-Premio Bond showed less microleakage scores than Clearfil SE Bond with different modes of application.

\section{Clinical Significance}

A major problem in cervical composite restorations is the presence of microleakage in the cervical wall. Different adhesive systems have been designed to overcome this problem.

\section{Acknowledgments}

Authors are grateful to Dental Research Institute of Dental School of Isfahan University of Medical Sciences, Iran. Source of funding is supported by Isfahan University of Medical Sciences.

\section{References}

1. Mitra SB, Wu D, Holmes BN. An application of nanotechnology in advanced dental materials. J Am Dent Assoc 2003;134(10):1382-1390. DOI: 10.14219/jada.archive.2003.0054.

2. Demarco FF, Corrêa MB, Cenci MS, et al. Longevity of posterior composite restorations: not only a matter of materials. Dent Mater 2012;28(1):87-101. DOI: 10.1016/j.dental.2011.09.003.

3. Korkmaz Y, Ozel E, Attar N, et al. Microleakage and scanning electron microscopy evaluation of all-in-one self-etch adhesives and their respective nanocomposites prepared by erbium: yttriumaluminum-garnet laser and bur. Lasers Med Sci 2010;25(4):493-502. DOI: 10.1007/s10103-009-0672-5.

4. Opdam N, Loomans B, Roeters F, et al. Five-year clinical performance of posterior resin composite restorations placed by dental students. J Dent 2004;32(5):379-383. DOI: 10.1016/j.jdent.2004.02.005.

5. Krmek SJ, Bogdan I, Simeon P, et al. A three-dimensional evaluation of microleakage of class $V$ cavities prepared by the very short pulse mode of the erbium: yttrium-aluminium-garnet laser. Lasers Med Sci 2010;25(6):823-828. DOI: 10.1007/s10103-009-0707-y.

6. Waldman G, Vaidyanathan T, Vaidyanathan J. Microleakage and resin-to-dentin interface morphology of pre-etching versus selfetching adhesive systems. Open Dent J 2008;2(1):120-125. DOI: 10.2174/1874210600802010120.

7. Baygin O, Korkmaz FM, Arslan I. Effects of different types of adhesive systems on the microleakage of compomer restorations in Class $\mathrm{V}$ cavities prepared by Er, Cr: YSGG laser in primary teeth. Dent Mater J 2012;31(2):206-214. DOI: 10.4012/dmj.2011-133.

8. Ritter AV, Swift EJ, Heymann HO, et al. An eight-year clinical evaluation of filled and unfilled one-bottle dental adhesives. J Am Dent Assoc 2009;140(1):28-37. DOI: 10.14219/jada.archive.2009.0015.

9. Manhart J, Neuerer P, Scheibenbogen-Fuchsbrunner A, et al. Threeyear clinical evaluation of direct and indirect composite restorations in posterior teeth. J Prosthet Dent 2000;84(3):289-296. DOI: 10.1067/ mpr.2000.108774.

10. Zhao X, Li S, Gu L, et al. Detection of marginal leakage of class v restorations in vitro by micro-computed tomography. Oper Dent 2014;39(2):174-180. DOI: 10.2341/12-182-L.

11. Geerts S, Bolette A, Seidel L, et al. An in vitro evaluation of leakage of two etch and rinse and two self-etch adhesives after thermocycling. Int J Dent 2012;2012:852841. DOI: 10.1155/2012/852841.

12. Ferrari M, Goracci G, García-Godoy F. Bonding mechanism of three "one-bottle" systems to conditioned and unconditioned enamel and dentin. Am J Dent 1997;10(5):224-230. PMID: 9522696.

13. Van Meerbeek B, Perdigao J, Lambrechts $P$, et al. The clinical performance of adhesives. J Dent 1998;26(1):1-20. DOI: 10.1016/s03005712(96)00070-x.
14. De Munck Jd, Van Landuyt K, Peumans M, et al. A critical review of the durability of adhesion to tooth tissue: methods and results. J Dent Res 2005;84(2):118-132. DOI: 10.1177/154405910508 400204.

15. Santini $A$, Ivanovic $V$, Ibbetson $R$, et al. Influence of marginal bevels on microleakage around Class $V$ cavities bonded with seven self-etching agents. Am J Dent 2004;17(4):257-261. PMID: 15478487.

16. Abo T, Uno S, Sano H. Comparison of bonding efficacy of an allin-one adhesive with a self-etching primer system. Eur J Oral Sci 2004;112(3):286-292. DOI: 10.1111/j.1600-0722.2004.00126.x.

17. Kubo S, Yokota $H$, Hayashi Y. Microleakage of cervical cavities restored with flowable composites. Am J Dent 2004;17(1):33-37. PMID: 15241907.

18. Santini A, Ivanovic $V$, Ibbetson $R$, et al. Influence of cavity configuration on microleakage around Class $\mathrm{V}$ restorations bonded with seven self-etching adhesives. J Esthet Restor Dent 2004;16(2):128-135. DOI: 10.1111/j.1708-8240.2004.tb00020.x.

19. Sano H, Yoshikawa T, Pereira P, et al. Long-term durability of dentin bonds made with a self-etching primer, in vivo. J Dent Res 1999;78(4):906-911. DOI: 10.1177/00220345990780041101.

20. Hayakawa T, Kikutake K, Nemoto K. Influence of self-etching primer treatment on the adhesion of resin composite to polished dentin and enamel. Dent Mater 1998;14(2):99-105. DOI: 10.1016/s01095641(98)00015-3.

21. Perdigao J, Swift E, Walter R. Fundamental concepts of enamel and dentin adhesion. In: Sturdevant's art and science of operative dentistry. 2014. p. 114-140. DOI:10.1016/B978-0-323-47833. 00005-8.

22. Schroeder M, Correa IC, Bauer J, et al. Influence of adhesive strategy on clinical parameters in cervical restorations: a systematic review and meta-analysis. J Dent 2017;62:36-53. DOI: 10.1016/j.jdent.2017. 05.006.

23. Khosravi K, Ataei E, Mousavi M, et al. Effect of phosphoric acid etching of enamel margins on the microleakage of a simplified all-in-one and a self-etch adhesive system. Oper Dent 2009;34(5):531-536. DOI: 10.2341/08-026-L.

24. Kermanshah $\mathrm{H}$, Khorsandian $\mathrm{H}$. Comparison of microleakage of Scotchbond ${ }^{T M}$ Universal Adhesive with methacrylate resin in Class $\checkmark$ restorations by two methods: Swept source optical coherence tomography and dye penetration. Dent Res J 2017;14(4):272. DOI: 10.4103/1735-3327.211651.

25. Gupta A, Tavane P, Gupta PK, et al. Evaluation of microleakage with total etch, self etch and universal adhesive systems in Class V restorations: an in vitro study. J Clin Diagn Res 2017;11(4):ZC53. DOI: 10.7860/JCDR/2017/24907.9680.

26. Khoroushi M, Shirban F, Shirban M. Marginal microleakage and morphological characteristics of a solvent-free one-step self-etch adhesive (B1SF). J Dent (Tehran) 2013;10(1):32-40. PMID: 23724201.

27. Tay FR, Carvalho R, Sano H, et al. Effect of smear layers on the bonding of a self-etching primer to dentin. J Adhes Dent 2000;2(2):99-116. PMID: 11317405.

28. Pereira P, Okuda M, Sano $H$, et al. Effect of intrinsic wetness and regional difference on dentin bond strength. Dent Mater 1999;15(1):46-53. DOI: 10.1016/s0109-5641(99)00013-5.

29. Carvalho RM, Tjäderhane L, Manso AP, et al. Dentin as a bonding substrate. Endod Topics 2009;21(1):62-88. DOI: 10.1111/j.16011546.2012.00274.x.

30. Van Meerbeek B, De Munck J, Yoshida Y, et al. Adhesion to enamel and dentin: current status and future challenges. Oper Dent 2003;28(3):215-235. PMID: 12760693.

31. Yoshida Y, Nagakane K, Fukuda R, et al. Comparative study on adhesive performance of functional monomers. J Dent Res 2004;83(6):454-458. DOI: 10.1177/154405910408300604.

32. Hannig M, Reinhardt K, Bott B. Self-etching primer vs phosphoric acid: an alternative concept for composite-to-enamel bonding. Oper Dent 1999;24(3):172-180. PMID: 10530279. 
33. Osorio R, Toledano M, De Leonardi G, et al. Microleakage and interfacial morphology of self etching adhesives in class $V$ resin composite restorations. J Biomed Materials Res Part B App Biomater 2003;66(1):399-409. DOI: 10.1002/jbm.b.10024.

34. Cardoso P, Placido E, Francci C, et al. Microleakage of Class V resinbased composite restorations using five simplified adhesive systems. Am J Dent 1999;12(6):291-294. PMID: 10850249.

35. Opdam N, Roeters F, Feilzer A, et al. Marginal integrity and postoperative sensitivity in Class 2 resin composite restorations in vivo. J Dent 1998;26(7):555-562. DOI: 10.1016/s0300-5712(97) 00042-0.

36. Takamizawa T, Barkmeier W, Tsujimoto A, et al. Effect of phosphoric acid pre-etching on fatigue limits of self-etching adhesives. Oper Dent 2015;40(4):379-395. DOI: 10.2341/13-252-L.
37. Choi A-N, Lee J-H, Son S, et al. Effect of dentin wetness on the bond strength of universal adhesives. Materials (Basel) 2017;10(11):1224. DOI: $10.3390 / m a 10111224$

38. Imai A, Takamizawa T, Sai K, et al. Influence of application method on surface free-energy and bond strength of universal adhesive systems to enamel. Eur J Oral Sci 2017;125(5):385-395. DOI: 10.1111/eos. 12361.

39. Szesz A, Parreiras S, Reis A, et al. Selective enamel etching in cervical lesions for self-etch adhesives: a systematic review and meta-analysis. J Dent 2016;53:1-11. DOI: 10.1016/j.jdent.2016.05.009.

40. Kearns JO, Barry JG, Fleming GJ. Cuspal deflection and cervical microleakage scores to determine the adhesive potential of universal bonding systems. J Dent 2014;42(8):970-976. DOI: 10.1016/j.jdent. 2014.05.013. 